\title{
CONTRIBUTIONS TO THE ANALYTIC THEORY OF $J$-FRACTIONS
}

\author{
BY \\ H. S. WALL AND MARION WETZEL \\ To Ernst Hellinger
}

1. Introduction. Continued fractions of the form

$$
\frac{1}{b_{1}+z}-\frac{a_{1}^{2}}{b_{2}+z}-\frac{a_{2}^{2}}{b_{3}+z}-\cdots \quad\left(a_{p} \neq 0\right),
$$

in which the coefficients $a_{p}, b_{p}$ are complex numbers, and $z$ is a complex parameter, have been called $J$-fractions because of their connection with the infinite matrices known as $J$-matrices. The theory of $J$-fractions with real coefficients includes the Stieltjes continued fraction theory and certain of its extensions. In a recent paper, Hellinger and Wall [3](1) treated the case where $a_{p}$ is real and $b_{p}$ is an arbitrary complex number with nonnegative imaginary part. In these cases, the $J$-fraction obviously has the property that all the quadratic forms

$$
\sum_{r=1}^{p} \Im\left(b_{r}+z\right) \xi_{r}^{2}-2 \sum_{r=1}^{p-1} \Im\left(a_{r}\right) \xi_{r} \xi_{r+1}, \quad p=1,2,3, \cdots,
$$

are positive definite for $\Im(z)>0$. In the present paper we investigate the general class of all $J$-fractions for which these quadratic forms are positive definite.

We develop a theory of these positive definite $J$-fractions analogous to the classical theory $\left({ }^{2}\right)$. The main points can be summarized as follows.

A. Nest of circles. Regarding the $J$-fraction as an infinite sequence of linear transformations, we construct for $\Im(z)>0$ a nest of circles $K_{p}(z)$ $(p=1,2,3, \cdots)$ lying in the lower half-plane, each contained in the preceding, and such that $f_{p}(z)$ lies on $K_{p}(z)$. A formula for the radius $r_{p}(z)$ of $K_{p}(z)$ is obtained which involves a value of one of the quadratic forms (1.2). Two cases have to be distinguished, according as $\lim _{p=\infty} r_{p}(z)=0$ ("limitpoint case") or $\lim _{p=\infty} r_{p}(z)>0$ ("limit-circle case").

B. Theorem of invariability. We show that the distinction between the two cases is invariant under a change in the particular value of the parameter $z$ in the upper half-plane. Furthermore, in the limit-point case the $J$-fraction converges and represents an analytic function of $z$ for $\Im(z)>0$.

Presented to the Society, April 23, 1943; received by the editors May 17, 1943.

(1) Numbers in square brackets refer to the bibliography at the end of the paper.

(2) We refer the reader to [3] for a summary of the history of these problems and for references. 
C. Asymptotic representation. A function $f(z)$ which for $\Im(z)>0$ is analytic and has its value in all the circles $K_{p}(z)$ is called an equivalent function of the $J$-fraction. We show that an arbitrary equivalent function $f(z)$ is represented asymptotically by the $J$-fraction in the sense $\left({ }^{3}\right)$ that

$$
\lim _{z=\infty} z^{2 p}\left(f(z)-f_{p}(z)\right)=0
$$

as $z$ approaches $\infty$ along any path for which $\Im(z) \geqq \delta>0$ ( $\delta$ an arbitrary positive number).

D. Stieltjes integral representation. We show that an arbitrary equivalent function $f(z)$ has a Stieltjes integral representation

$$
f(z)=\int_{-\infty}^{+\infty} \frac{d \phi(u)}{z+u}
$$

where $\phi(u)$ is a bounded nondecreasing function.

Further developments of the positive definite $J$-fraction and connections with other problems in the analytic theory of continued fractions are contained in the paper Quadratic forms and convergence regions for continued fractions appearing in the Duke Mathematical Journal.

2. Positive definite $J$-fractions. A $J$-fraction is a continued fraction of the form

$$
\frac{1}{b_{1}+z}-\frac{a_{1}^{2}}{b_{2}+z}-\frac{a_{2}^{2}}{b_{3}+z}-\cdots \quad\left(a_{p} \neq 0\right)
$$

in which $a_{1}, a_{2}, a_{3}, \cdots$ are arbitrary complex numbers different from zero, $b_{1}, b_{2}, b_{3}, \cdots$ are arbitrary complex numbers, and $z=x+i y$ is a complex variable. We denote its $p$ th approximant by

$$
f_{p}(z)=A_{p}(z) / B_{p}(z) \quad(p=1,2,3, \cdots),
$$

where the $A_{p}(z)$ and $B_{p}(z)$ are given by the recursion formulas

$$
\begin{aligned}
& a_{p} A_{p}(z)=\left(b_{p}+z\right) A_{p-1}(z)-a_{p-1} A_{p-2}(z), \\
& a_{p} B_{p}(z)=\left(b_{p}+z\right) B_{p-1}(z)-a_{p-1} B_{p-2}(z),
\end{aligned}
$$

with the initial values

$$
\begin{array}{ll}
A_{0}(z)=0, & A_{1}(z)=a_{1}^{-1}, \\
B_{0}(z)=1, & B_{1}(z)=a_{1}^{-1}\left(b_{1}+z\right) .
\end{array}
$$

From the recursion formulas we have immediately the determinant formula:

$$
A_{p}(z) B_{p-1}(z)-A_{p-1}(z) B_{p}(z)=a_{p}^{-1} .
$$

(3) This definition differs somewhat from that of Hellinger and Wall [3, p. 122]. They restrict the path of $z$ to lie in an angular region: $\alpha \leqq \arg z \leqq \pi-\alpha, 0<\alpha<\pi / 2$. 
With the continued fraction (2.1) we associate the bilinear form

If we put

$$
H_{p}(\xi, \eta)=\sum_{r=1}^{p} \Im\left(b_{r}+z\right) \xi_{r} \eta_{r}-\sum_{r=1}^{p-1} \Im\left(a_{r}\right)\left(\xi_{r} \eta_{r+1}+\xi_{r+1} \eta_{r}\right) .
$$

$$
\alpha_{r}=\Im\left(a_{r}\right), \quad \beta_{r}=\Im\left(b_{r}\right),
$$

then the discriminant of $(2.5)$ is

$$
D_{p}(y)=\left|\begin{array}{ccccc}
\beta_{1}+y, & -\alpha_{1}, & 0, & \cdots, & 0 \\
-\alpha_{1}, & \beta_{2}+y, & -\alpha_{2}, & \cdots, & 0 \\
\cdot, & \cdot, & \cdot, & \cdots, & . \\
\cdot, & \cdot, & \cdot, & \cdots, & \cdot \\
0, & \cdots, & -\alpha_{p-2}, & \beta_{p-1}+y, & -\alpha_{p-1} \\
0, & \cdots, & 0, & -\alpha_{p-1}, & \beta_{p}+y
\end{array}\right| .
$$

There is an extensive theory of $J$-fractions in which the coefficients $a_{p}, b_{p}$ are all real. Recently this theory has been extended to the case where the $a_{p}$ are real and the $b_{p}$ are complex numbers with nonnegative imaginary parts. In these cases it will be observed that the quadratic forms $H_{p}(\xi, \xi)$ are positive definite for $\Im(z)>0$. This suggests that we consider the general class of all $J$-fractions for which these quadratic forms are positive definite. Accordingly, we make the following definition:

DefinITION 2.1. The J-fraction (2.1) is called positive definite if the quadratic forms $H_{p}(\xi, \xi), p=1,2,3, \cdots$, are positive definite for all values of $z$ for which $\Im(z)>0$, that is

$$
D_{p}(y)>0 \text { for } y>0, \quad p=1,2,3, \cdots .
$$

The fundamental property of positive definite $J$-fractions, and one which forms the basis of the present investigation, is given by the following theorem:

THEOREM A. If the J-fraction (2.1) is positive definite, then its approximants $f_{p}(z)$ lie in the lower half-plane for all values of $z$ in the upper half-plane:

$$
\Im\left(f_{p}(z)\right)<0 \text { for } \Im(z)>0, \quad p=1,2,3, \cdots \text {. }
$$

Proof. When (2.7) holds, then the determinants

$$
D_{s}^{(r)}(y)=\left|\begin{array}{ccccc}
\beta_{1+r}+y, & -\alpha_{1+r}, & 0, & \cdots, & 0 \\
-\alpha_{1+r}, & \beta_{2+r}+y, & -\alpha_{2+r}, & \cdots, & 0 \\
\cdot, & \cdot, & \cdot, & \cdots, & \cdot \\
\cdot, & \cdot, & \cdot, & \cdots, & \cdot \\
0, & \cdots, & \alpha_{s-2}, & \beta_{s-1}+y, & -\alpha_{s-1} \\
0, & \ldots, & 0, & -\alpha_{s-1}, & \beta_{s}+y
\end{array}\right|>0
$$


for $s=1,2,3, \cdots, r=1,2,3, \cdots, s-1$. We write $D_{s}(y)$ for $D_{s}^{(0)}(y)$. Now

$$
\Im\left(f_{1}(z)\right)=-\left(\beta_{1}+y\right) /\left|b_{1}+z\right|^{2}<0,
$$

inasmuch as $D_{1}(y)=\beta_{1}+y>0$. If we put $t_{p}=\Re\left(b_{p}+z\right), s_{p}=\Re\left(a_{p}\right)$, and $b_{1}^{*}(z)=1 / f_{2}(z)$, then

$$
\Im\left(b_{1}^{*}(z)\right)=\frac{\left[\left(\beta_{1}+y\right) t_{2}^{2}+\left(\beta_{2}+y\right) s_{1}^{2}-2 \alpha_{1} t_{2} s_{1}\right]+\left(\beta_{2}+y\right) D_{2}}{\left|b_{2}+z\right|^{2}}>0,
$$

inasmuch as $\beta_{2}+y>0, D_{2}(y)>0$, and the expression in the brackets is a value of the positive definite form $\mathrm{H}_{2}(\xi, \xi)$.

Using induction, let us assume that the conditions

$$
\begin{aligned}
& D_{1}(y)>0 \\
& D_{2}(y)>0, D_{2}^{(1)}(y)>0, \\
& . . . . . . . . . \\
& D_{n}(y)>0, D_{n}^{(1)}(y)>0, \cdots, D_{n}^{(n-1)}(y)>0
\end{aligned}
$$

imply that $\Im\left(f_{p}(z)\right)<0, p=1,2,3, \cdots, n$, and we shall prove that these conditions together with

$$
D_{n+1}^{(r)}(y)>0, \quad r=0,1,2, \cdots, n,
$$

imply that $\Im\left(f_{n+1}(z)\right)<0$.

We have

$$
f_{n+1}(z)=\frac{1}{b_{1}+z}-\frac{a_{1}^{2}}{b_{2}+z}-\cdots-\frac{a_{n-2}^{2}}{b_{n-1}+z}-\frac{a_{n-1}^{2}}{b_{n}^{*}(z)},
$$

where

$$
b_{n}^{*}(z)=b_{n}+z-a_{n}^{2} /\left(b_{n+1}+z\right) .
$$

Under the hypothesis of the induction, it suffices to show that when the relations (2.12) hold, then the relations obtained from these by replacing $\beta_{n}+y$ by $\beta_{n}^{*}(z)=\Im\left(b_{n}^{*}(z)\right)$ also hold. Inasmuch as $\beta_{n}+y$ appears only in the determinants of the last row of the inequalities (2.12), we need consider only the determinants $D_{n}^{*(r)}(z), r=0,1,2, \cdots, n-1$, obtained from $D_{n}^{(r)}(y)$ by replacing $\beta_{n}+y$ by $\beta_{n}^{*}(z)$.

We find immediately that

$$
D_{n}^{*(r)}(z)=\frac{\left[D_{n}^{(r)} t_{n+1}^{2}+\left(\beta_{n+1}+y\right) D_{n-1}^{(r)} s_{n}^{2}-2 \alpha_{n} D_{n-1}^{(r)} t_{n+1} s_{n}\right]+\left(\beta_{n+1}+y\right) D_{n+1}^{(r)}}{\left|b_{n+1}+z\right|^{2}}
$$

where $D_{n-1}^{(n-1)}(y)$ must be set equal to unity. When (2.12) and (2.13) hold we therefore conclude that $(2.15)$ is positive inasmuch as the determinant of the 
quadratic form in the brackets is

$$
D_{n-1}^{(r)}(y) \cdot D_{n+1}^{(r)}(y)>0 .
$$

In obtaining this we have used the recursion formula

$$
D_{n+1}^{(r)}(y)=\left(\beta_{n+1}+y\right) D_{n}^{(r)}-\alpha_{n}^{2} D_{n-1}^{(r)}(y) .
$$

We shall conclude this section with a series of lemmas which will be used later.

LEMMA 2.1. If $B_{p}(z)$ is the pth denominator of the positive definite J-fraction (2.1), then

$$
\Im\left(a_{p+1} B_{p+1} \bar{B}_{p}\right)=\sum_{r=1}^{p+1}\left(\beta_{r}+y\right)\left|B_{r-1}\right|^{2}-\sum_{r=1}^{p} \alpha_{r}\left(B_{r} \bar{B}_{r-1}+\bar{B}_{r} B_{r-1}\right) .
$$

Hence, since $B_{0}=1$, then $\Im\left(a_{p+1} B_{p+1} \bar{B}_{p}\right)>0$ for $\Im(z)=y>0$, and therefore

$$
B_{p}(z) \neq 0 \text { for } \Im(z)>0, \quad p=0,1,2, \ldots \text {. }
$$

Proof. By (2.2) we have:

$$
\begin{aligned}
& a_{1} B_{1}=\left(b_{1}+{ }^{\circ} z\right) B_{0}, \\
& a_{2} B_{2}+a_{1} B_{0}=\left(b_{2}+z\right) B_{1} \text {, } \\
& a_{p+1} B_{p+1}+a_{p} B_{p-1}=\left(b_{p+1}+z\right) B_{p} .
\end{aligned}
$$

Multiplying these equations by $\bar{B}_{0}, \bar{B}_{1}, \cdots, \bar{B}_{p}$, respectively, adding, and then taking the imaginary parts of the resulting sums, we obtain (2.18).

LEMMA 2.2. For the function

$$
\sigma_{p}(y)=\alpha_{p}^{2} D_{p-1}(y) / D_{p}(y),
$$

we have the following relations:

$$
\begin{gathered}
\sigma_{p}(y)=\frac{\alpha_{p}^{2}}{\beta_{p}+y-\sigma_{p-1}(y)}, \quad p=2,3, \cdots, \sigma_{1}(y)=\alpha_{1}^{2} /\left(\beta_{1}+y\right) ; \\
\sigma_{p}\left(y_{1}\right) \geqq \sigma_{p}\left(y_{2}\right) \quad \text { if } \quad 0<y_{1}<y_{2} \\
0 \leqq \sigma_{p-1}(y)<\beta_{p}+y .
\end{gathered}
$$

Consequently, the limit

$$
\lim _{y \rightarrow+0} \sigma_{p}(y)=\sigma_{p}(+0)
$$

exists and is finite. 
Proof. The determinants $D_{p}(y)$ satisfy the identity:

$$
D_{p}(y)=\left(\beta_{p}+y\right) D_{p-1}(y)-\alpha_{p-1}^{2} D_{p-2}(y) \text {. }
$$

Hence we have

$$
\sigma_{p}(y)=\frac{\alpha_{p}^{2}}{\left(D_{p}(y) / D_{p-1}(y)\right)}=\frac{\alpha_{p}^{2}}{\beta_{p}+y-\alpha_{p-1}^{2} D_{p-2}(y) / D_{p-1}(y)},
$$

which is (2.20); then (2.21) follows immediately from (2.20) by mathematical induction; and (2.22) follows from (2.24), written in the form

$$
\sigma_{p-1}(y)=\beta_{p}+y-D_{p}(y) / D_{p-1}(y) \text {. }
$$

LEMMA 2.3. The limiting values $\sigma_{p}(+0)$ satisfy the relations:

$$
\begin{gathered}
\beta_{p+1}+y>\sigma_{p}(+0) ; \\
\sigma_{p}(+0)>\frac{\alpha_{p}^{2}}{\beta_{p}+y-\sigma_{p-1}(+0)}, \text { if } \alpha_{p} \neq 0 .
\end{gathered}
$$

Proof. This lemma follows immediately from (2.22) and (2.20).

LEMMA 2.4. Let

$$
v_{p}(x+i y ; t)=B_{p-1}(x+i y)-\alpha_{p}\left(D_{p-1}(t) / D_{p}(t)\right) B_{p}(x+i y) .
$$

If $0<t<y$, then

$$
0 \leqq\left|v_{p}(x+i t ; t)\right|<\left|v_{p}(x+i y ; t)\right|
$$

and

$$
0<\left|B_{p}(x+i t)\right|<\left|B_{p}(x+i y)\right| .
$$

Proof. The expression $v_{p}(z ; t), z=x+i y$, is the denominator of the modified approximant

$$
\frac{1}{b_{1}+z}-\frac{a_{1}^{2}}{b_{2}+z}-\cdots-\frac{a_{p-1}^{2}}{b_{p}+z-c}, \quad c=\frac{a_{p}}{\left(\alpha_{p} D_{p-1}(t) / D_{p}(t)\right)} .
$$

This may be regarded as obtained from $B_{p}(z)$ by replacing $b_{p}$ by $b_{p}-c$, and therefore the determinant $D_{p}(y)$ becomes

$$
D_{p}(y)-D_{p-1}(y) D_{p}(t) / D_{p-1}(t) .
$$

This is positive for $0<t<y$, by (2.25) and (2.21). Consequently, by Lemma $2.1, v_{p}(x+i y ; t) \neq 0$ if $y>t>0$, that is, the polynomial in $z, v_{p}(z ; t)$, has all its zeros in the half-plane $\Im(z) \leqq t$. By $(2.19), B_{p}(z)$ has all its zeros in the halfplane $\Im(z) \leqq 0$. The inequalities (2.28) and (2.29) are immediate consequences, if we regard the polynomials as products of the vectors from the point $z$ to their zeros. 
3. The nest of circles. In the preceding section we showed that a $J$-fraction has all its approximants $f_{p}(z)$ in the lower half-plane, for $y=\Im(z)>0$, provided all the determinants $D_{p}(y)$ are positive for $y$ positive. Our next problem is to determine more specifically the location of the approximants. Inasmuch as the $J$-fraction may be regarded as a sequence of linear transformations, the considerations used in similar problems $\left({ }^{4}\right)$ suggest that a nest of circular regions $H_{p}(z)$ can be obtained such that $f_{p}(z)$ lies in $H_{p}(z)$. Such nests of circular regions can be obtained in various ways.

In general, it is desirable that a nest of circular regions $H_{p}(z)$ have the following properties:

I. $f_{p}(z)$ is on the circle $K_{p}(z)$ bounding $H_{p}(z)$;

II. $H_{p}(z)$ is a proper circular region;

III. $H_{p}(z)$ contains $H_{p+1}(z), p=1,2,3, \cdots$.

The first property is desirable in order that a simple formula can be obtained for the radius $r_{p}(z)$ of $K_{p}(z)$; the second is desirable in order that one can obtain bounds for the values of the approximants; the third is desirable in order that there will be two and only two cases, namely: the regions $H_{p}(z)$ have one and only one point in common ("limit-point case"), or the regions $H_{p}(z)$ have a circular region in common ("limit-circle case"). This last leads to a fourth desired property:

IV. The distinction between the two cases is invariant under a change in the particular value of the parameter $z$ in the upper half-plane.

We shall now proceed to construct a nest of circles having these properties. Consider the linear transformation

$$
t=t_{p}(w ; z)=\frac{1}{b_{1}+z}-\frac{a_{1}^{2}}{b_{2}+z}-\cdots-\frac{a_{p-1}^{2}}{b_{p}+z-}-\frac{a_{p}^{2}}{w}
$$

of the $w$-plane into the $t$-plane. In view of the condition for positive definiteness, it is natural to require that $w$ satisfy the inequality

$$
\left|\begin{array}{ccccc}
\beta_{1}+y, & -\alpha_{1}, & 0, & \cdots, & 0 \\
-\alpha_{1}, & \beta_{2}+y, & -\alpha_{2}, & \cdots, & 0 \\
\cdot, & \cdot, & \cdot, & \cdots & . \\
\cdot, & \cdot \cdot & \cdot, & \cdots, & \cdot \\
0, & \cdots, & -\alpha_{p-1}, & \beta_{p}+y, & -\alpha_{p} \\
0, & \cdots, & 0, & -\alpha_{p}, & \Im(w)
\end{array}\right|>0,
$$

that is,

$$
\Im(w)>\sigma_{p}(y) \text { where } \sigma_{p}(y)=\alpha_{p}^{2} D_{p-1}(y) / D_{p}(y),
$$

(4) Cf., for instance, Weyl [5, 6], Hamburger [1], Hellinger [2], R. Nevanlinna [4], Hellinger and Wall [3]. 
and then to determine the corresponding range of $t$ as our proposed region $H_{p}(z)$. In the special case $p=1$ we find that the range of $t$ is in general a circular region, but that for particular values of $z(\Im(z)>0)$ it may be a half-plane. Thus the second of the four requirements listed above is not satisfied. In order to make it possible to obtain smaller circular regions, we shall replace (3.2) by an arbitrary half-plane

$$
\Im(w)>\tau_{p}(y),
$$

where

$$
\tau_{p}(y) \geqq \sigma_{p}(y), \text { for } y>0 .
$$

The function $\tau_{p}(y)$ will be appropriately determined later on.

We next obtain formulas for the center $C_{p}$ and radius $r_{p}$ of the circle $K_{p}$ into which the straight line $\Im(w)=\tau_{p}(y)$ is carried by the transformation (3.1). We first write (3.1) in the form

$$
t=t_{p}(w ; z)=\frac{A_{p}(z) w-a_{p} A_{p-1}(z)}{B_{p}(z) w-a_{p} B_{p-1}(z)},
$$

and recall that $B_{p}(z)$ and $B_{p-1}(z)$ are different from zero for $\Im(z)>0$. The transformation $t=t_{p}(w)$ maps the point $w=a_{p} B_{p-1}(z) / B_{p}(z)$ into the point $t=\infty$. Hence, inasmuch as the center $t=C_{p}$ of $K_{p}(z)$ can be produced by inversion of $t=\infty$ in $K_{p}$, then $C_{p}$ must correspond under this transformation to the reflection of the point

$$
w=a_{p} B_{p-1}(z) / B_{p}(z)
$$

in the line $\Im(w)=\tau_{p}(y)$. Since the required reflection of the point (3.6) is

$$
\bar{a}_{p} \bar{B}_{p-1}(z) / \bar{B}_{p}(z)+2 i \tau_{p}(y)
$$

we therefore have

$$
C_{p}=\frac{A_{p}(z)\left(\bar{a}_{p} \bar{B}_{p-1}(z) / \bar{B}_{p}(z)+2 i \tau_{p}(y)\right)-a_{p} A_{p-1}(z)}{B_{p}(z)\left(\bar{a}_{p} \bar{B}_{p-1}(z) / \bar{B}_{p}(z)+2 i \tau_{p}(y)\right)-a_{p} B_{p-1}(z)} .
$$

Inasmuch as $t_{p}(\infty)=A_{p}(z) / B_{p}(z)$ is on $K_{p}(z)$, the radius $r_{p}$ of $K_{p}$ is given by

$$
r_{p}=\left|\frac{A_{p}(z)}{B_{p}(z)}-C_{p}\right|=\frac{1}{\left.2\left|\Im\left(\bar{a}_{p} B_{p}(z) \bar{B}_{p-1}(z)\right)+\tau_{p}(y)\right| B_{p}(z)\right|^{2} \mid}
$$

By (2.18) and the equality

$$
\Im\left(a_{p+1} B_{p+1} \bar{B}_{p}\right)=\left(\beta_{p+1}+y\right)\left|B_{p}\right|^{2}+\Im\left(\bar{a}_{p} \bar{B}_{p-1} B_{p}\right) \quad\left(B_{p}=B_{p}(z)\right),
$$

which follows from the recursion formulas, we get: 


$$
\begin{aligned}
\Im\left(\bar{a}_{p} B_{p} \bar{B}_{p-1}\right)+\tau_{p}(y)\left|B_{p}\right|^{2}= & \tau_{p}(y)\left|B_{p}\right|^{2}+\sum_{r=1}^{p}\left(\beta_{r}+y\right)\left|B_{r-1}\right|^{2} \\
& -\sum_{r=1}^{p} \alpha_{r}\left(B_{r} \bar{B}_{r-1}+\bar{B}_{r} B_{r-1}\right) .
\end{aligned}
$$

This can be written in the forms

$$
\begin{aligned}
& \Im\left(\bar{a}_{p} B_{p} \bar{B}_{p-1}\right)+\tau_{p}(y)\left|B_{p}\right|^{2}=\left(\tau_{p}(y)-\sigma_{p}(y)\right)\left|B_{p}\right|^{2} \\
& \quad+\sum_{r=1}^{p+1}\left(\beta_{r}+y\right)\left|B_{r-1}\right|^{2}-\sum_{r=1}^{p} \dot{\alpha}_{r}\left(B_{r} \bar{B}_{r-1}+\bar{B}_{r} B_{r-1}\right)-\frac{D_{p+1}(y)}{D_{p}(y)}\left|B_{p}\right|^{2}
\end{aligned}
$$

and

$$
\begin{aligned}
\Im\left(\bar{a}_{p} B_{p} \bar{B}_{p-1}\right)+\tau_{p}(y)\left|B_{p}\right|^{2}= & \left(\tau_{p}(y)-\sigma_{p}(y)\right)\left|B_{p}\right|^{2} \\
& +\sum_{r=1}^{p} \frac{D_{r}(y)}{D_{r-1}(y)}\left|B_{r-1}-\alpha_{r} \frac{D_{r-1}(y)}{D_{r}(y)} B_{r}\right|^{2} .
\end{aligned}
$$

If $\alpha_{p}=0$ the last formula shows that $r_{p}(z)$ is finite; if $\alpha_{p} \neq 0$, then $r_{p}(z)$ is finite if

$$
\tau_{p}(y)>\sigma_{p}(y) \quad \text { for } y>0 .
$$

Formula (3.12) shows simultaneously that the point (3.7), which is mapped into $C_{p}$, lies in the interior of the half-plane $\Im(w)>\tau_{p}(y)$, so that this halfplane is mapped into the interior of $H_{p}(z)$.

If $\alpha_{p} \neq 0$, then by Lemma 2.2 the function $\sigma_{p}(y)$ is a bounded monotone decreasing function of $y$ which has a finite limit $\sigma_{p}(+0)$ as $y$ approaches +0 ; if $\alpha_{p}=0$, then $\sigma_{p}(y) \equiv 0$. If now we take

$$
\tau_{p}(y) \equiv \sigma_{p}(+0),
$$

the inequality (3.3) will hold and, moreover, (3.13) will hold if $\alpha_{p} \neq 0$. The nest of circular regions $H_{p}(z)$ corresponding to this value of $\tau_{p}(y)$ clearly has Property I and Property II. We consider next the third property:

PROPERTY III. The nest of circular regions $H_{p}(z)$ with centers and radii given by (3.8) and (3.9) in which $\tau_{p}(y)=\sigma_{p}(+0)$ has the property that $H_{p}(z)$ contains $H_{p+1}(z), p=1,2,3, \cdots$.

Proof. We show first that $H_{p+1}(z)$ and $H_{p}(z)$ have a region in common. In fact, as previously noted, the $(p+1)$ th approximant, $f_{p+1}(z)$, is upon $K_{p+1}(z)$. Hence, it suffices to show that $f_{p+1}(z)$ is on the interior of $H_{p}(z)$. This is equivalent to the statement that $\Im\left(b_{p+1}+z\right)=\beta_{p+1}+y>\sigma_{p}(+0)$, which is true by (2.26). To complete the proof, we now show that the boundaries $K_{p+1}(z)$ and $K_{p}(z)$ can have at most one point in common. That is, there can be at most one pair of values $w_{p+1}$ and $w_{p}$ such that 


$$
\Im\left(w_{p+1}\right)=\sigma_{p+1}(+0), \quad \Im\left(w_{p}\right)=\sigma_{p}(+0),
$$

and such that $t_{p}\left(w_{p}\right)=t_{p+1}\left(w_{p+1}\right)$, or

$$
w_{p}=b_{p+1}+z-a_{p+1}^{2} / w_{p+1} .
$$

Taking imaginary parts and setting $\Im\left(w_{p}\right)=\sigma_{p}(+0), w_{p+1}=u+i \sigma_{p+1}(+0)$ ( $u$ real), and $s_{p+1}=\Re\left(a_{p+1}\right)$, we find that $u$ must satisfy the quadratic equation:

$$
\begin{aligned}
& {\left[\beta_{p+1}+y-\sigma_{p}(+0)\right] u^{2}-2 s_{p+1} \alpha_{p+1} u} \\
& \quad+\left[\left(\beta_{p+1}+y-\sigma_{p}(+0)\right) \sigma_{p+1}^{2}(+0)+\left(s_{p+1}^{2}-\alpha_{p+1}^{2}\right) \sigma_{p+1}(+0)\right]=0 .
\end{aligned}
$$

If $\alpha_{p+1}=0$, so that $\sigma_{p+1}(+0)=0$, this equation has only the solution $u=0$. Thus $w_{p+1}=0, w_{p}=\infty$, and $K_{p+1}(z)$ is tangent to $K_{p}(z)$. If $\alpha_{p+1} \neq 0$, the quadratic equation (3.14) has no real solution, inasmuch as the discriminant is equal to

$$
\begin{aligned}
{\left[\alpha_{p+1}^{2}-\sigma_{p+1}(+0)\left(\beta_{p+1}+y-\sigma_{p}(\right.\right.} & +0))] \\
& \cdot\left[s_{p+1}^{2}+\sigma_{p+1}(+0)\left(\beta_{p+1}+y-\sigma_{p}(+0)\right)\right],
\end{aligned}
$$

which is negative by (2.27). Hence, in this case, $K_{p+1}(z)$ and $K_{p}(z)$ can have no point in common, and Property III is established.

We have shown that the nest of circles has the first three of the desired properties. Furthermore, we find that the center and radius of the first circle $K_{1}(z)$ are:

$$
\begin{aligned}
& C_{1}=\frac{2 \alpha_{1}\left(\alpha_{1}\left(t_{1}+x\right)-s_{1} \beta_{1}\right)-i\left(\beta_{1} \alpha_{1}^{2}+\beta_{1} s_{1}^{2}+2 \alpha_{1}^{2} y\right)}{2\left[y\left(\alpha_{1}^{2}\left(\beta_{1}+y\right)+\beta_{1} s_{1}^{2}\right)+\left(\alpha_{1}\left(t_{1}+x\right)-\beta_{1} s_{1}\right)^{2}\right]}, \\
& r_{1}=\frac{\beta_{1} s_{1}^{2}+\beta_{1} \alpha_{1}^{2}}{2\left[y\left(\alpha_{1}^{2}\left(\beta_{1}+y\right)+\beta_{1} s_{1}^{2}\right)+\left(\alpha_{1}\left(t_{1}+x\right)-\beta_{1} s_{1}\right)^{2}\right]},
\end{aligned}
$$

where $a_{1}=s_{1}+i \alpha_{1}$, and $b_{1}=t_{1}+i \beta_{1}$. It follows that $r_{1} \leqq\left|\Im\left(C_{1}\right)\right|$, and hence the circles lie in the lower half-plane. Moreover, these formulas show that for all $z$ such that $y=\Im(z) \geqq \delta>0$, the circles lie in a finite region depending only upon $\delta$. Thus, there exists a finite number $M_{\delta}$ such that

$$
|\mathrm{t}| \leqq M_{\delta}, \text { for } t \text { in } H_{p}(z) \text { and } \Im(z) \geqq \delta \quad(p=1,2, \cdots) .
$$

If the $a_{p}$ are real, then each circle is tangent to the preceding, the first being tangent to the real axis from below at the origin, and the positive definite $J$-fraction reduces to that treated by Hellinger and Wall [3]. If the $a_{p}$ are not real, then each circle is contained entirely within the preceding, the first circle having an arbitrary location in the lower half-plane. In the accompany- 
ing figure, the first three circles $K_{p}(1+i)$ are shown for the positive definite $J$-fraction

$$
\frac{1}{z+i}+\frac{1}{z+i}-\frac{1}{z+i}+\frac{1}{z+i}-\cdots
$$

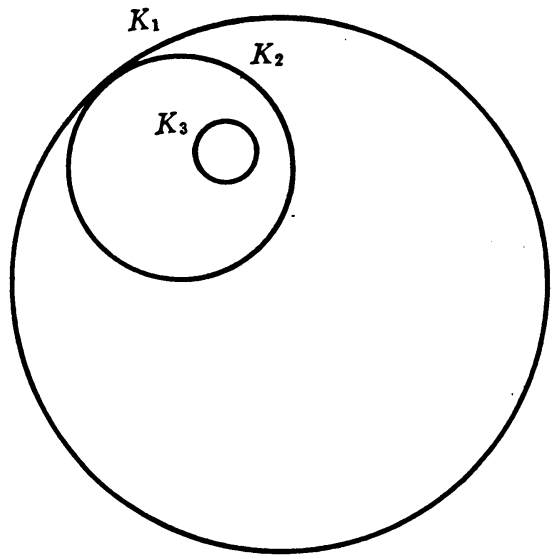

The centers, radii, and values of $\sigma_{p}(+0)$ are as follows:

$$
\begin{array}{lll}
\sigma_{1}=1, & C_{1}=(1 / 3)-(1 / 2) i, & r_{1}=1 / 6 ; \\
\sigma_{2}=0, & C_{2}=(1 / 4)-(7 / 16) i, & r_{2}=1 / 16 ; \\
\sigma_{3}=2 / 3, & C_{3}=(15 / 53)-(91 / 212) i, & r_{3}=3 / 212 .
\end{array}
$$

This example illustrates the location of the circles for a general positive definite $J$-fraction.

The following section contains the proof that the nest of circles has Property IV.

4. The theorem of invariability. Since the circular regions $H_{p}(z)$ are all finite, and $H_{p}(z) \supset H_{p+1}(z)$, there are two cases to be distinguished for a particular value of the parameter $z$ :

Case I. The limit-point case. The circular regions $H_{p}(z)$ have one and only one point $f(z)$ in common; the radius $r_{p}(z)$ of $K_{p}(z)$ converges to zero for $p=\infty$; the expression

$$
\begin{aligned}
& {\left[\sigma_{p}(+0)-\sigma_{p}(y)\right]\left|B_{p}(z)\right|^{2}} \\
& \quad+\sum_{q=1}^{p} \frac{D_{q}(y)}{D_{q-1}(y)}\left|B_{q-1}(z)-\alpha_{q} \frac{D_{q-1}(y)}{D_{q}(y)} B_{q}(z)\right|^{2}
\end{aligned}
$$

tends to the limit $\infty$ as $p$ approaches $\infty$.

Case II. The limit-circle case. The circular regions $H_{p}(z)$ have a circular 
region in common; the radius $r_{p}(z)$ of $K_{p}(z)$ has a positive limit for $p=\infty$; the expression (4.1) has a finite limit for $p=\infty$.

In Case I the $J$-fraction converges to the value $f(z)$. In Case II the $J$-fraction may or may not converge.

We shall now show that this distinction between the two cases is invariant under a change in the particular value of the parameter $z$ in the upper halfplane.

Theorem B (The Theorem of Invariability). If Case I holds for one value of $z$ in the upper half-plane, $\Im(z)>0$, then Case I holds for every value of $z$ in the upper half-plane, $\Im(z)>0$. That is, if for $p=\infty$ the expression (4.1) has the limit $+\infty$ for one value of $z$ with $\Im(z)>0$, then it has the limit $+\infty$ for every value of $z$ with $\Im(z)>0$.

Proof. Suppose that as $p$ approaches $\infty$ the expression (4.1) tends to $\infty$ for $z=x+i t, t>0$. Then, by the inequalities (2.28) and (2.29), it follows that for $y>t$,

$$
\begin{aligned}
\lim _{p=\infty}\{ & \left(\sigma_{p}(+0)-\sigma_{p}(t)\right)\left|B_{p}(x+i y)\right|^{2} \\
& \left.\quad+\sum_{q=1}^{p} \frac{D_{q}(t)}{D_{q-1}(t)}\left|B_{q-1}(x+i y)-\alpha_{q} \frac{D_{q-1}(t)}{D_{q}(t)} B_{q}(x+i y)\right|^{2}\right\}=\infty .
\end{aligned}
$$

Now, by (3.10), the expression in the braces can be written as

$$
\begin{aligned}
\sum_{q=1}^{p}\left(\beta_{q}+t\right)\left|B_{q-1}(x+i y)\right|^{2}-\sum_{q=1}^{p} \alpha_{q}\left[B_{q}(x+i y) \bar{B}_{q-1}(x+i y)\right. \\
\left.+\bar{B}_{q}(x+i y) B_{q-1}(x+i y)\right]+\sigma_{p}(+0)\left|B_{p}(x+i y)\right|^{2} .
\end{aligned}
$$

Consequently, if $t$ is replaced by $y$, this expression must increase, and therefore (4.2) holds for $t=y$.

This shows that if the expression (4.1) tends to $\infty$ for $z=x+i t, t>0$, it tends to $\infty$ for $z=x+i y, y>t$. That is, the limit-point case holds along any vertical segment $S$ from $z=x+i t$ to $z=x+i y, y>t$.

Let $\zeta, \Im(\zeta)>0$, be an arbitrary point in the upper half-plane. Let $G$ be a bounded closed simply-connected region in $\Im(z)>0$, containing $\zeta$ and the segment $S$ on the interior. We shall prove that if $L_{1}$ and $L_{2}$ are any two points common to all the regions $H_{p}(\zeta)$, then $L_{1}=L_{2}$, and therefore we shall have the limit-point case for $z=\zeta$. We can select two sequences of points $u_{p}$ and $v_{p}$ such that

$$
\Im\left(u_{p}\right) \geqq \sigma_{p}(+0), \quad \Im\left(v_{p}\right) \geqq \sigma_{p}(+0), \quad t_{p}\left(u_{p}\right)=L_{1}, \quad t_{p}\left(v_{p}\right)=L_{2} .
$$

The sequences of rational functions of $z$ : 


$$
t_{p}\left(u_{p}\right)=\frac{A_{p}(z) u_{p}-a_{p} A_{p-1}(z)}{B_{p}(z) u_{p}-a_{p} B_{p-1}(z)}, \quad t_{p}\left(v_{p}\right)=\frac{A_{p}(z) v_{p}-a_{p} A_{p-1}(z)}{B_{p}(z) v_{p}-a_{p} B_{p-1}(z)}
$$

are uniformly bounded over $G$ by virtue of (3.15). Therefore, we may select subsequences from $\left\{t_{p}\left(u_{p}\right)\right\}$ and $\left\{t_{p}\left(v_{p}\right)\right\}$, converging uniformly over $G$ to analytic limits $f_{1}(z)$ and $f_{2}(z)$, respectively. Inasmuch as $f_{1}(x+i y) \equiv f_{2}(x+i y)$ for $y \geqq t$ along the segment $S$, it follows that $f_{1}(z) \equiv f_{2}(z)$ for $\Im(z)>0$, and, in particular,

$$
L_{1}=f_{1}(\zeta)=f_{2}(\zeta)=L_{2}
$$

as was to be proved.

5. The reciprocals of the $J$-matrix. The matrix

$$
\begin{gathered}
J=\left(k_{p q}\right), \text { where } k_{p p}=b_{p}+z, k_{p, p+1}=k_{p+1, p}=-a_{p}, \\
k_{p q}=0 \text { if }|p-q|>1,
\end{gathered}
$$

formed with the coefficients of the $J$-fraction (2.1), is called a $J$-matrix. In this section we shall investigate the symmetrical reciprocals, $\left(\rho_{p q}\right)$, of the $J$-matrix, which are given by the formula $[3$, p. 116]:

$$
\text { (5.1) } \rho_{p q}=\rho_{p q}(z)=\left\{\begin{array}{c}
B_{p-1}(z) B_{q-1}(z)\left[f(z)-\frac{A_{q-1}(z)}{B_{q-1}(z)}\right], \quad p=1,2, \cdots, q, \\
B_{p-1}(z) B_{q-1}(z)\left[f(z)-\frac{A_{p-1}(z)}{B_{p-1}(z)}\right], \\
p=q+1, q+2, q+3, \cdots,
\end{array}\right.
$$

where $f(z)$ is an arbitrary function. We shall show that if for $\Im(z)>0$ the value of $f(z)$ lies in all the circles $K_{p}(z)$, then the quadratic forms

$$
R_{n}(\xi, \xi)=\sum_{p, q=1}^{n} \rho_{p q} \xi_{p} \xi_{q}, \quad n=1,2,3, \cdots,
$$

satisfy a certain boundedness condition. This will enable us in $\$ 6$ to obtain asymptotic and integral expressions for the $J$-fraction.

The $\rho_{p q}$ satisfy the recursion formulas

$$
-a_{p-1} \rho_{p-1, q}+\left(b_{p}+z\right) \rho_{p q}-a_{p} \rho_{p+1, q}=\delta_{p, q}=\left\{\begin{array}{lll}
0 & \text { if } & p \neq q, \\
1 & \text { if } & p=q,
\end{array}\right.
$$

where $a_{0}=1, \rho_{0, q}=0, \rho_{11}=f(z)$.

Let $\xi_{1}, \xi_{2}, \xi_{3}, \cdots$ be real numbers with $\xi_{q}=0$ for $q>n$, where $n$ is an arbitrary fixed positive integer. On multiplying (5.3) by $\xi_{q}$ and summing over $q$, we then obtain

$$
-a_{p-1} \eta_{p-1}+\left(b_{p}+z\right) \eta_{p}-a_{p} \eta_{p+1}=\xi_{p}
$$


where

$$
\eta_{p}=\sum_{q=1}^{n} \rho_{p q} \xi_{q}
$$

From (5.1) we see that $\rho_{n+1, q} / \rho_{n q}$ is independent of $q$ for $q=1,2,3, \cdots, n$. Moreover, if we put $w_{n}=a_{n} \rho_{n, q} / \rho_{n+1, q}$, then

so that, by (3.5),

$$
w_{n}=a_{n} \frac{B_{n-1}(z) f(z)-A_{n-1}(z)}{B_{n}(z) f(z)-A_{n}(z)}
$$

$$
f(z)=t_{n}\left(w_{n} ; z\right)=t_{n-1}\left(b_{n}+z-a_{n}^{2} / w_{n} ; z\right) .
$$

Consequently, the function $f(z)$ has its value in the circle $K_{n-1}(z)$ if and only if

$$
\Im\left(b_{n}+z-a_{n}^{2} / w_{n}\right) \geqq \sigma_{n-1}(+0),
$$

or

$$
\begin{aligned}
\Im\left(a_{n}^{2} / w_{n}\right) & \leqq \beta_{n}+y-\sigma_{n-1}(+0) \\
& =D_{n}(y) / D_{n-1}(y)-\left(\sigma_{n-1}(+0)-\sigma_{n-1}(y)\right) .
\end{aligned}
$$

Now

$$
a_{n} \rho_{n+1, q}=\left(a_{n}^{2} / w_{n}\right) \rho_{n, q},
$$

or, on multiplying by $\xi_{q}$ and summing over $q$, we have

$$
a_{n} \eta_{n+1}=\left(a_{n}^{2} / w_{n}\right) \eta_{n}
$$

We now multiply (5.4) by $\bar{\eta}_{p}$, sum over $p$ from 1 to $n$, and then eliminate the quantity $a_{n} \eta_{n+1} \bar{\eta}_{n}$ by means of (5.7). This gives the relation:

$$
\sum_{p=1}^{n}\left(b_{p}+z\right)\left|\eta_{p}\right|^{2}-\sum_{p=1}^{n-1} a_{p}\left(\eta_{p} \bar{\eta}_{p+1}+\eta_{p+1} \bar{\eta}_{p}\right)=\frac{a_{n}^{2}}{w_{n}}\left|\eta_{n}\right|^{2}+\sum_{p=1}^{n} \xi_{p} \bar{\eta}_{p} .
$$

If we consider only the imaginary part and make use of the inequality (5.6), we then have the relation (cf. (3.12)):

$$
\begin{aligned}
\sum_{p=1}^{n-1} \frac{D_{p}(y)}{D_{p-1}(y)}\left|\eta_{p}-\alpha_{p} \frac{D_{p-1}(y)}{D_{p}(y)} \eta_{p+1}\right|^{2} & +\left[\sigma_{n-1}(+0)-\sigma_{n-1}(y)\right]\left|\eta_{n}\right|^{2} \\
& +\sum_{p=1}^{n} \xi_{p} \Im\left(\eta_{p}\right) \leqq 0
\end{aligned}
$$

We note in passing that if $f(z)=t$ is regarded as a complex variable, and if we take $\xi_{1}=1, \xi_{p}=0$ for $p>1$, the inequality (5.8) becomes an inequality defining our nest of circular regions. We remark that the presence of the term $\left[\sigma_{n-1}(+0)-\sigma_{n-1}(y)\right]\left|\eta_{n}\right|^{2}$ prevents our using the method of Hellinger [2, 
p. 23] to show that one circle is contained in the preceding ("Property III"). We consider now the system of equations

$$
\begin{aligned}
& \theta_{1}=\frac{1}{D_{0}(y)}-\alpha_{1} \frac{\eta_{2}}{D_{1}(y)} \\
& \theta_{2}=\quad \frac{\eta_{2}}{D_{1}(y)}-\alpha_{2} \frac{\eta_{3}}{D_{2}(y)}, \\
& \theta_{n-1}=\quad \frac{\eta_{n-1}}{D_{n-2}(y)}-\alpha_{n-1} \frac{\eta_{n}}{D_{n-1}(y)}, \\
& \theta_{n}=\quad \frac{\eta_{n}}{D_{n-1}(y)} .
\end{aligned}
$$

This system may conveniently be written in matrix form as

$$
\theta=\Gamma \eta,
$$

where $\Gamma$ is the matrix of the system and $\theta, \eta$ are one-column matrices. These equations may be solved for $\eta_{1}, \eta_{2}, \cdots, \eta_{n}$ in terms of $\theta_{1}, \theta_{2}, \cdots, \theta_{n}$, and on using the resulting values in (5.8), that inequality becomes

$$
\begin{aligned}
\sum_{p=1}^{n-1} D_{p}(y) D_{p-1}(y)\left|\theta_{p}\right|^{2} & +\left[\sigma_{n-1}(+0)-\sigma_{n-1}(y)\right] D_{n-1}^{2}(y)\left|\theta_{n}\right|^{2} \\
& +\sum_{p=1}^{n} \xi_{p} \Im\left(\eta_{p}\right) \leqq 0
\end{aligned}
$$

or, if we put

$$
E_{p}(y)=\left\{\begin{array}{l}
D_{p}(y) D_{p-1}(y), \text { for } p=1,2 \cdots, n-1, \\
{\left[\sigma_{n-1}(+0)-\sigma_{n-1}(y)\right] D_{n-1}^{2}(y), \text { for } p=n,}
\end{array}\right.
$$

then

$$
\sum_{p=1}^{n} E_{p}(y)\left|\theta_{p}\right|^{2}+\sum_{p=1}^{n} \xi_{p} \Im\left(\eta_{p}\right) \leqq 0
$$

The $E_{p}(y)$ are polynomials in $y ; E_{p}(y)$ is of degree $2 p-1$ for $p \leqq n-1 ; E_{n}(y)$ is of degree $2 n-2$ if $\alpha_{n-1} \neq 0$, and is identically equal to zero if $\alpha_{n-1}=0$. Moreover, $E_{p}(y)>0$ for $y>0(p=1,2,3, \cdots, n-1)$ and $E_{n}(y)>0$ for $y>0$ if $\alpha_{n-1} \neq 0$.

Turning now to the quadratic form (5.2), we have, by (5.5) and (5.9),

$$
R_{n}(\xi, \xi)=\sum_{p=1}^{n} \xi_{p} \eta_{p}=\xi^{\prime} \eta=\left(\xi^{\prime} \Gamma^{-1}\right) \theta=\zeta^{\prime} \theta=\sum_{p=1}^{n} \zeta_{p} \theta_{p}
$$


where

$$
\zeta^{\prime}=\left(\xi^{\prime} \Gamma^{-1}\right) \quad \text { or } \quad \zeta=\left(\Gamma^{-1}\right)^{\prime} \xi .
$$

The $\zeta_{p}$ are real inasmuch as the $\xi_{p}$ and the coefficients in the matrix $\Gamma$ are all real. We shall agree that $\xi_{n}=0$ if $\alpha_{n-1}=0$, in which case $\eta_{n}$ and $\zeta_{n}$ will not appear in (5.12). By (5.11) and Schwarz's inequality we then have

$$
\begin{aligned}
\left|R_{n}(\xi, \xi)\right|^{2}=\left|\sum_{p=1}^{n} \frac{\zeta_{p}}{E_{p}^{1 / 2}(y)} E_{p}^{1 / 2}(y) \theta_{p}\right|^{2} & \leqq \sum_{p=1}^{n} \frac{\zeta_{p}^{2}}{E_{p}(y)} \cdot \sum_{p=1}^{n} E_{p}(y)\left|\theta_{p}\right|^{2} \\
& \leqq \sum_{p=1}^{n} \frac{\zeta_{p}^{2}}{E_{p}(y)}\left[-\sum_{p=1}^{n} \xi_{p} \Im\left(\eta_{p}\right)\right] .
\end{aligned}
$$

Thus,

$$
\left|R_{n}(\xi, \xi)\right|^{2} \leqq \sum_{p=1}^{n} \frac{\zeta_{p}^{2}}{E_{p}(y)} \cdot \Im\left[-R_{n}(\xi, \xi)\right] \leqq \sum_{p=1}^{n} \frac{\zeta_{p}^{2}}{E_{p}(y)} \cdot\left|R_{n}(\xi, \xi)\right|,
$$

and therefore

$$
\left|R_{n}(\xi, \xi)\right| \leqq \sum_{p=1}^{n} \frac{\zeta_{p}^{2}}{E_{p}(y)}
$$

It should be emphasized that in case $\alpha_{n-1}=0$, then $\xi_{n}=0$ and the last summation runs only to $n-1$. This result may be formulated in the following theorem:

TheOREM C (TheOREM OF BOUNDEDNEss). Consider the quadratic form (5.2) in which $\rho_{p q}$ is given by formula (5.1), where $f(z)$ is an arbitrary function whose values for $\Im(z)>0$ lie in the circle $K_{n-1}(z)$. Let $\xi_{1}, \cdots, \xi_{n}$ be arbitrary real numbers, except that $\xi_{n}=0$, if $\alpha_{n-1}=0$. Then $R_{n}(\xi$, $\xi)$ satisfies the inequality (5.14), where the $\zeta_{p}$ are given in terms of the $\xi_{p}$ by (5.13), and the $E_{p}(y)$ are polynomials in y given by (5.10).

If the $\alpha_{p}$ are all zero, then (5.14) reduces to the " $E$-boundedness" of Hellinger and Wall $[3$, p. 117].

We shall use this theorem to obtain some estimates for the $\rho_{p q}$ which will be used in the next section. Let $\left(\Gamma^{-1}\right)^{\prime}=\left(g_{p q}\right)$. We observe that for a given $q$ and arbitrary $p, g_{p q}$ is a constant multiple of $D_{q-1}(y)$, and is equal to zero for $p<q$. If now, for any $p<n$, we let $\xi_{p}=1$, and $\xi_{q}=0$ for $q \neq p$, the inequality (5.14) reduces to

$$
\left|\rho_{p p}\right| \leqq \sum_{r=p}^{n} \frac{g_{r p}^{2}}{E_{r}(y)} .
$$

Consequently, taking in to account the degree of $E_{r}(y)$ (cf. (5.10)) and of the $g_{r p}$ as just defined, we see that $\rho_{p p}(z)=O(1 / y)$ for $y>0$. Next, we let $\xi_{p}=\xi_{q}=1$ 
for any $p$ and $q$ for which $p<q<n, \xi_{r}=0$ for $r \neq p, q$, and (5.14) becomes

$$
\left|\rho_{p p}+2 \rho_{p q}+\rho_{q q}\right| \leqq \sum_{r=p}^{n} \frac{\left(g_{r p}+g_{r q}\right)^{2}}{E_{r}(y)}
$$

so the $\rho_{p p}+2 \rho_{p q}+\rho_{q q}=O(1 / y)$, and therefore, since $n$ was arbitrary, we have:

$$
\rho_{p q}=O(1 / y) \text { for } y>0 \quad(p, q=1,2,3, \cdots) .
$$

Finally, by the recursion formula (5.3),

$$
\rho_{p q}=\frac{a_{p-1} \rho_{p-1, q}-b_{p} \rho_{p, q}+a_{p} \rho_{p+1, q}}{z}+\frac{\delta_{p q}}{z}
$$

from which we conclude immediately by $(5.15)$ that

$$
\rho_{p q}(z)=\delta_{p q} / z+G_{p q}(z) / y z, \quad\left|G_{p q}(z)\right|<M \quad \text { for } y>0,
$$

where $M$ is a constant, independent of $z$; and also,

$$
\rho_{p+r, p}(z)=O\left(1 / z^{r+1}\right) \text { for } y=\Im(z)>0 .
$$

6. Asymptotic and integral expressions for the $J$-fraction. If $\left\{w_{p}\right\}$ is any sequence of constants such that $\Im\left(w_{p}\right) \geqq \sigma_{p}(+0)(p=1,2,3, \cdots)$, then the sequence $\left\{t_{p}\left(w_{p} ; z\right)\right\}$ of generalized approximants of the positive definite $J$-fraction (2.1) is uniformly bounded over every region of the upper halfplane whose distance from the real axis is positive. Hence, this sequence contains a subsequence which converges for $\Im(z)>0$ to an analytic limit $f(z)$, and the value of $f(z)$ lies in every circle $K_{p}(z)$. In the limit-point case there is defined in this way one and only one analytic function, namely, the value of the $J$-fraction. In the limit-circle case there are infinitely many such analytic functions. These functions, and any function $f(z)$ which for $\Im(z)>0$ is analytic and has its value in the circle $K_{p}(z)$ for $p=1,2,3, \cdots$, will be called equivalent to the $J$-fraction. We shall show that in the limit-point case, and also in the limit-circle case, an equivalent function $f(z)$ is represented asymptotically by the $J$-fraction in accordance with the following theorem.

THEOREM D. An arbitrary equivalent function $f(z)$. is asymptotically equal to the positive definite $J$-fraction in the sense that the expression

$$
z^{2 p}\left(f(z)-A_{p}(z) / B_{p}(z)\right) \quad(p=1,2,3, \cdots)
$$

approaches zero as. $z$ approaches infinity along any path lying in the half-plane $\Im(z) \geqq \delta$, where $\delta$ is an arbitrary positive number.

Proof. From (5.1) we obtain:

$$
z^{2 p}\left(f(z)-A_{p}(z) / B_{p}(z)\right)=z^{2 p} \rho_{p+1 ; p} / B_{p}(z) B_{p-1}(z) .
$$


By (5.17) it follows that this expression is of the order of $1 / z$, and therefore has the limit 0 as $z$ tends to $\infty$ along any path in the half-plane $\Im(z) \geqq \delta>0$, as was to be proved.

We consider now the problem of obtaining Stieltjes integral representations for the equivalent functions. In view of the fact that (5.16) with $p=q=1$ gives for an arbitrary equivalent function $f(z)$ the estimate

$$
f(z)=1 / z+G(z) / z \Im(z), \quad|G(z)|<M, \quad \Im(z)>0,
$$

we may apply a result of Hellinger and Wall [3, p. 123], and obtain immediately the following theorem.

THEOREM E. An arbitrary equivalent function $f(z)$ of the positive definite $J$-fraction (2.1) has a Stieltjes integral representation of the form

$$
f(z)=\int_{-\infty}^{+\infty} \frac{d \phi(u)}{z+u}, \quad \phi(+\infty)-\phi(-\infty)=1,
$$

where $\phi(u)$ is a bounded, nondecreasing function.

In the preceding approach to the problem of obtaining integral representations for the equivalent functions, the rôle of the rational fractions, as approximants for the functions, has not been emphasized. This is in contrast with the work of Stieltjes, where the approximants were used directly in obtaining the integrals. It is interesting to see that even for the general positive definite $J$-fraction considered in this paper, the transition from the approximants to the integrals can be made in a way comparable with that of Stieltjes. This fact is a consequence of the following theorem.

THEOREM F. Let $R(z)$ be a rational function of $z$ satisfying the following two conditinns:

$$
\begin{aligned}
& \text { (i) } \lim _{z \rightarrow \infty} z R(z)=k, \quad k \text { a real positive number; } \\
& \text { (ii) } \Im(R(z))<0, \text { for } \Im(z)>0 \text {. }
\end{aligned}
$$

Then, there exists a nondecreasing function $\phi(u)$ such that $0 \leqq \phi(u) \leqq k$ and

$$
R(z)=\int_{-\infty}^{+\infty} \frac{d \phi(u)}{z+u} .
$$

Proof. From (ii) it readily follows that all the poles of $R(z)$ must lie in the lower half-plane, $\Im(z) \leqq 0$, and that any real pole must be simple with positive residue. Therefore, $R(z)$ must have the form

$$
R(z)=\sum_{s=1}^{r} \sum_{t=1}^{m_{s}} \frac{(-1)^{t-1} p_{t}^{(s)}}{\left(z+a_{s}\right)^{t}}+\sum_{s=1}^{h} \frac{M_{s}}{z+x_{s}},
$$


where $x_{s}$ is real, $M_{s}$ is real and positive, $p_{t}^{(s)}$ is a constant, and $-a_{s}, \Im\left(a_{s}\right)>0$, is a nonreal pole of multiplicity $m_{s}$. From (i) it follows that

$$
p_{1}^{(1)}+p_{1}^{(2)}+\cdots+p_{1}^{(r)}+M_{1}+M_{2}+\cdots+M_{h}=k .
$$

We prove the existence of the real integral

$$
\begin{aligned}
\frac{1}{2 \pi i} \int_{-\infty}^{+\infty}(\bar{R}(-u) & -R(-u)) d u \\
= & \frac{1}{2 \pi i} \int_{-\infty}^{+\infty} \sum_{s=1}^{r} \sum_{t=1}^{m_{s}}\left(\frac{p_{t}^{(s)}}{\left(u-a_{s}\right)^{t}}-\frac{p_{t}^{(s)}}{\left(u-\bar{a}_{s}\right)^{t}}\right) d u .
\end{aligned}
$$

Let the denominator of $R(z)$ (supposed irreducible) be of degree $q+h$. Then, the denominator of the rational function of $u$ in the integrand of (6.6) is $2 q$, and the numerator is of degree at most $2 q-1$. Indeed, the numerator is of degree less than $2 q-1$, so that the integral converges absolutely. For, the coefficient of $u^{2 q-1}$ in the numerator is

$$
\sum_{s=1}^{r} p_{1}^{(s)}-\sum_{s=1}^{r} p_{1}^{(s)}
$$

which is 0 by (6.5) inasmuch as $k$ is real by hypothesis. Therefore, by (ii) we see that the function

$$
\phi_{1}(u)=\frac{1}{2 \pi i} \int_{-\infty}^{u}(\bar{R}(-t)-R(-t)) d t
$$

is a nonnegative nondecreasing function of $u$; and, by the theory of residues,

$$
\phi_{1}(+\infty)=p_{1}^{(1)}+p_{1}^{(2)}+\cdots+p_{1}^{(r)} .
$$

Let $\phi_{2}(u)$ be a step-function such that $\phi_{2}(-\infty)=0$, having the saltus $M_{8}$ at $u=-x_{s}$, so that

$$
\int_{-\infty}^{+\infty} \frac{d \phi_{2}(u)}{z+u}=\sum_{s=1}^{h} \frac{M_{s}}{z+x_{s}}
$$

and write $\phi(u)=\phi_{1}(u)+\phi_{2}(u)$. Then, $\phi(u)$ is nondecreasing and $0 \leqq \phi(u) \leqq k$. Moreover, again using the theory.of residues, we get (6.3).

If we have a sequence $\left\{f_{p}(z)\right\}$ of rational functions of $z$ satisfying the conditions (i) and (ii), where $k$ in (i) is independent of the particular function of the sequence being considered, then we see at once that the sequence is uniformly bounded over any region of $z$ such that $\Im(z) \geqq \delta>0$ :

$$
\left|f_{p}(z)\right| \leqq k / \delta, \quad p=1,2,3, \cdots \text {. }
$$

This is true in particular for the sequence of approximants of a positive defi- 
nite $J$-fraction. Therefore, one may apply to the sequence the well known theorems on uniformly bounded families of analytic functions, for example, the Stieltjes-Vitali theorem. Furthermore, the associated sequence of monotone functions must contain a subsequence converging to a monotone limitfunction; and it is permissible to take the limit under both the integral and the differential sign.

\section{REFERENCES}

1. H. Hamburger, Über eine Erweiterung des Stieltjesschen Momentenproblems. I, II, and III, Math. Ann. vol. 81 (1920) pp. 235-319; vol. 82 (1920) pp. 120-164 and 168-187.

2. E. Hellinger, Zur Stieltjesschen Kettenbruchtheorie, Math. Ann. vol. 86 (1922) pp. 18-29.

3. E. Hellinger and H. S. Wall, Contributions to the analytic theory of continued fractions and infinite matrices, Ann. of Math. (2) vol. 44 (1943) pp. 103-127.

4. R. Nevanlinna, Asymptotische Entwicklungen beschränkter Funktionen und das Stieltjessche Momenten problem, Annales Academiae Fennicae, A, vol. 18 (1922) no. 5.

5. $\mathrm{H}$. Weyl, Über gewöhnliche Differentialgleichungen mit Singularitäten und die zugehörigen Entwicklungen willkürlicher Funktionen, Math. Ann. vol. 68 (1910) pp. 220-269.

6. - Über das Pick-Nevanlinna'sche Interpolationsproblem und sein infinitesimales Analogon, Ann. of Math. (2) vol. 36 (1935) pp. 230-254.

NORTHWESTERN UNIVERSITY,

Evanston, Ill. 